Olivia LúCia Nunes COSTA ${ }^{1}$

Eliane Menezes Flores Santos ${ }^{2}$

EDUARDo Martins NetTo ${ }^{3}$

\section{Aspectos epidemiológicos e obstétricos de mulheres com perdas recorrentes da gravidez em uma maternidade pública do Nordeste do Brasil}

\author{
Epidemiological and obstetrics aspects in women with recurrent \\ pregnancy losses at a public maternity in the Brazilian Northeast
}

Artigo Original

Palavras-chave
Aborto habitual/epidemiologia
Cuidado pré-natal
Sobrepeso
Fatores de risco
Keywords
Abortion, habitual/epidemiology
Prenatal care
Overweight
Risk factors

Palavras-chave

\section{Resumo}

OBJETIVO: Descrever as características epidemiológicas e obstétricas de mulheres com perdas gestacionais de repetição. MÉTODOS: Estudo descritivo e analítico, que teve como critério de inclusão mulheres atendidas no ambulatório de perdas gestacionais de repetição (grupo de perdas), entre janeiro de 2006 e dezembro de 2010 . Foram excluídas as pacientes que não residiam em Salvador, na Bahia, e aquelas que não puderam ser contatadas por telefone. $\bigcirc$ Grupo Controle foi constituído por 204 gestantes de baixo risco ao pré-natal, atendidas entre maio de 2007 e abril de 2008. Foram excluídas desse grupo aquelas que não aceitaram participar da entrevista e com risco obstétrico. As variáveis pesquisadas foram: idade, escolaridade, ocupação, estado civil, etilismo, índice de massa corpórea e, como antecedentes obstétricos, a idade da gestação em que ocorreram as perdas. Para a análise estatística, utilizou-se o programa SPSS, versão 18.0. As médias e os desvios padrão das variáveis contínuas foram comparados utilizando-se o teste t de Student, já para as frequências das variáveis nominais, aplicou-se o teste do $\chi^{2}$. RESULTADOS: A média de idade das mulheres de perdas foi mais elevada do que no Controle $(32,3 \pm 6,3$ versus $26,5 \pm 6,4$ anos; $p<0,01)$. Houve predomínio do consumo de bebidas alcoólicas no grupo de perdas $(36,9$ versus $22,1 \%$; $p=0,01)$, assim como no estado civil $(93,2$ versus $66,7 \% ; p<0,011$, no qual elas eram casadas ou viviam em união estável, respectivamente. $\bigcirc$ índice de massa corpórea pré-gestacional foi superior no grupo de perdas (26,9 versus 23,5\%; $p<0,01)$. Nos antecedentes obstétricos, 103 mulheres com perdas gestacionais relataram 334 gestações. Destas, 56 tiveram dois ou mais abortos no primeiro trimestre e em 31 delas, duas ou mais gestações evoluíram para abortos tardios/prematuros extremos. CONCLUSÕES: Mulheres com perdas recorrentes incluídas neste estudo tiveram alguns fatores de risco identificados, tais como a idade mais avançada e o índice de massa corpórea superior. As observações são coerentes com as propostas mais recentes a respeito de perdas recorrentes que consideram a inclusão das perdas em várias idades gestacionais.

\section{Abstract}

PURPOSE: To describe the epidemiologic and obstetric characteristics of women with recurrent miscarriages. METHODS: A descriptive and analytical study whose inclusion criterion was every woman that was attended at the clinic for recurrent miscarriage (loss group), between January 2006 and December 2010. Patients that did not live in Salvador, Bahia, Brazil, and those who were not reached by telephone or whose number was not included in the medical record were not included. The Control Group consisted of 204 pregnant women seen at the low-risk prenatal care unit between May 2007 and April 2008. Women who did not accept to be interviewed and those with obstetric risk were excluded from the Control Group. The analyzed variables were: age, education, occupation, marital status, alcohol consumption, body mass index, obstetric history and the gestational age when the losses occurred. The SPSS 18.0 program was used for statistical analysis. Means and standard deviations of continuous variables were compared using the Student's t.test and the frequencies of the nominal variables were compared by the $\chi^{2}$ test. RESULTS: The mean age of women in the loss group was higher than in the Control Group (32.3 \pm 6.3 versus $26.5 \pm 6.4$ years old, $p<0.01$ ). Consumption of alcoholic beverages predominated in the loss group ( 36.9 versus $22.1 \%, p=0.01$ ), as well as marital

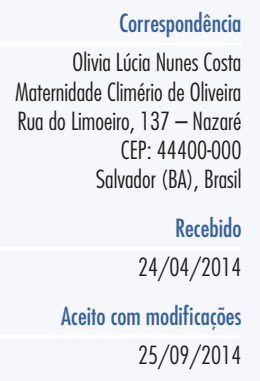

Correspondência Lúcia Nunes Cost Maternidade Climério de Oliveira CEP: 44400-000 Salvador (BA), Brasil

Recebido

modificações

25/09/2014
Departamento de Ginecologia, Obstetrícia e Reprodução Humana da Faculdade de Medicina da Bahia da Universidade Federal da Bahia - UFBA - Salvador (BA), Brasil.

'Departamento de Ginecologia, Obstetrícia e Reprodução Humana da Faculdade de Medicina da Bahia, Universidade Federal da Bahia UFBA - Salvador (BA), Brasil.

²Disciplina de Obstetrícia, Escola Bahiana de Medicina e Saúde Pública - EBMSP - Salvador (BA), Brasil.

${ }^{3}$ Hospital Universitário Professor Edgard Santos, Universidade Federal da Bahia - UFBA - Salvador (BA), Brasil.

Conflito de interesses: não há. 
status $(93.2$ versus $66.7 \%$ were married or living in a stable union, $\mathrm{p}<0.01)$. The pre-pregnancy body mass index was higher in the loss group (26.9 versus $23.5 \%, p<0.01$ ). Regarding obstetric history, 103 women with recurrent miscarriage reported 334 pregnancies. Fifty-six of them had 2 or more miscarriages in the first quarter and in 31 of them, 2 or more pregnancies progressed to late abortions/extremely preterm infants. CONCLUSIONS: Some risk factors were identified in women with recurrent losses, such as more advanced age and higher body mass index. These observations agree with more recent proposals regarding recurrent losses that consider the inclusion of losses in various gestational ages.

\section{Introdução}

A perda gestacional recorrente da gravidez ou o abortamento de repetição são definidos como a interrupção espontânea e consecutiva de três ou mais gestações, antes da $22^{\mathrm{a}}$ semana $^{1,2}$. As perdas de repetição ocorrem em até $5,0 \%$ das mulheres na fase reprodutiva ${ }^{3}$ e têm efeitos devastadores sobre a vida da mulher e da família. O conceito contempla a maior parte dos casos, uma vez que os abortamentos de repetição ocorrem predominantemente no primeiro trimestre da gestação $0^{4-6}$. Em definição recente, a Associação Americana de Medicina Reprodutiva $^{7}$ refere-se a duas ou mais perdas, sem mencionar a idade gestacional, o que traduz melhor, pela sua possível abrangência, a realidade dos ambulatórios, nos quais chegam mulheres com história de perdas nas mais variadas idades gestacionais.

$\mathrm{O}$ atendimento a mulheres com perdas gestacionais de repetição, sejam precoces ou tardias, representa um desafio, em função dos múltiplos fatores envolvidos na etiologia dos insucessos gestacionais. O obstetra, não raramente, esgota seus recursos propedêuticos, excluindo as causas mais frequentemente associadas às perdas, como as genéticas, anatômicas, hormonais e imunológicas, particularmente a síndrome de antifosfolípides ${ }^{8}$. Mas ainda assim, cerca de metade dos casos são classificados como causas desconhecidas ${ }^{3}$. Tão instigante constatação dá ensejo a investigações no sítio da implantação embrionária, como a expressão de proteínas relacionadas à receptividade do tecido endometrial ${ }^{9}$, e muitas outras envolvendo cromossomos $^{10,11}$ e polimorfismos em genes relacionados às trombofilias hereditárias ${ }^{12-15}$.

Por outro lado, considerando a obesidade como um problema de saúde pública na atualidade e a gestação como um dos fatores clássicos de desencadeamento e manutenção dessa condição ${ }^{16,17}$, estudos têm relatado efeitos adversos na evolução e nos resultados gestacionais, destacando-se maior risco para pré-eclâmpsia e macrossomia ${ }^{18-20}$. Em relação às mulheres com perdas gestacionais, está evidenciado ${ }^{21} \mathrm{o}$ aumento do risco de aborto em gestação subsequente nas obesas. O risco é acrescido pela idade da mulher e pelo número de perdas prévias. Um estudo ${ }^{22}$ relata que mulheres com obesidade e baixo peso apresentam maiores riscos de abortamento quando comparadas àquelas com sobrepeso e normais.

Resultados discordantes têm sido observados em estudos referentes a perdas gestacionais provenientes de vários continentes, e isso se deve não somente às características de cada população, mas sobretudo à seleção da amostra, por vezes incluindo abortamentos precoces, tardios ou mesmo insucessos nas gestações nos segundos e terceiros trimestres, como na definição de morbidade obstétrica da síndrome de antifosfolípides ${ }^{8,23}$. A dificuldade na interpretação e na comparação dos resultados leva os obstetras a indicarem a utilização de terapêuticas, por vezes sem eficácia comprovada, movidos pela ansiedade das próprias mulheres ${ }^{17}$. Em contrapartida, o conhecimento de que as perdas sucessivas tendem a ocorrer na mesma idade gestacional das precedentes ${ }^{7}$, conquanto represente a época de maior temor para as pacientes, permite a tomada de providências favoráveis ao êxito reprodutivo. Um estudo recente $^{24}$ enfoca as metodologias usadas na abordagem do tema e suas respectivas vantagens e desvantagens, enfatizando o planejamento dos estudos e evitando fatores de confusão, uma vez que o diagnóstico das perdas gestacionais é baseado numa série de eventos passados, cuja validade depende da qualidade das informações obtidas.

Com o intuito de avaliar o êxito gestacional num Ambulatório de Perdas Gestacionais de Repetição, verificou-se a necessidade da análise dos aspectos epidemiológicos, bem como o detalhamento dos antecedentes obstétricos, para melhor compreensão e comparação dos resultados, uma vez que esse conhecimento representa o primeiro passo na elucidação diagnóstica e conduta na gestação subsequente.

\section{Métodos}

Foi realizado um estudo descritivo e analítico, que se baseou em entrevista com as pacientes e consulta aos prontuários na Maternidade Climério de Oliveira da Universidade Federal da Bahia. Os critérios de inclusão no estudo foram: mulheres com antecedentes de perdas gestacionais de repetição, atendidas no ambulatório especializado entre janeiro de 2006 e dezembro de 2010. Foram excluídas aquelas que não residiam em Salvador. Foram listadas 310 mulheres e excluídas também aquelas cujo contato telefônico foi frustrado após 3 tentativas, assim distribuídas: 92 tinham mudado de número telefônico $(28,3 \%), 85$ não atenderam $(25,9 \%)$ e, em 30 casos, não constava o número no prontuário médico $(9,1 \%)$. Foram incluídas neste estudo 103 mulheres com perdas gestacionais de repetição, das quais 43 eram gestantes e 60 procuraram avaliação no serviço antes de tentarem uma nova gestação. 
O Grupo Controle foi constituído de 204 gestantes, atendidas de maio de 2007 a abril de 2008, em um pré-natal de baixo risco na mesma maternidade. Foram selecionadas as mulheres que aceitaram participar do estudo e excluídas aquelas que apresentaram algum risco obstétrico, as quais foram encaminhadas para atendimento especializado. A escolha desses ambulatórios não levou a um viés de seleção. No período de estudo, o número de nascidos vivos na Maternidade Climério de Oliveira da Universidade Federal da Bahia foi de 15.359 crianças. Muitas parturientes, porém, não haviam realizado pré-natal na instituição. Foram atendidas 6.408 mulheres nos ambulatórios (de baixo e alto risco, perdas gestacionais, diabetes e outras endocrinopatias, hipertensão arterial e falcemia), sendo que a maioria era para acompanhamento durante o pré-natal.

As seguintes variáveis foram consideradas: idade (em anos completos); raça (autorreferida pelas pacientes com perdas gestacionais e definida com interferência do observador nas gestantes de baixo risco); escolaridade (em três categorias: fundamental, médio e superior); ocupação (com ou sem renda); estado civil (as pacientes foram agrupadas em solteiras e em regime de união estável/casadas) e etilismo (consumo foi de, pelo menos, nove doses por semana) ${ }^{25}$. Para o índice de massa corpórea pré-gestacional (IMC), utilizou-se a fórmula de Quetelet (peso em quilogramas/quadrado da altura em metro ${ }^{26}$. O diagnóstico de sobrepeso/obesidade foi determinado pelo critério da Organização Mundial de Saúde (OMS) para cálculo do IMC, ou seja: normal - 18,5 a $24,9 \mathrm{~kg} / \mathrm{m}^{2}$; sobrepeso - 25 a $29,9 \mathrm{~kg} / \mathrm{m}^{2}$ e obeso maior que $30 \mathrm{~kg} / \mathrm{m}^{2}$.

Quanto aos aspectos obstétricos, consideraram-se mulheres com perdas gestacionais de repetição aquelas que tiveram dois ou mais insucessos consecutivos, independente da idade gestacional, ocorridos de forma espontânea e com comprovação diagnóstica hormonal e/ou ultrassonográfica da gestação. Para os antecedentes de perdas gestacionais, considerou-se a duração da gestação em 3 categorias: primeiro trimestre, até 13 semanas e 6 dias; segundo, entre 14 e 27 semanas e 6 dias e terceiro, entre 28 semanas e o termo da gestação.

Para a análise estatística, o programa Statistical Package for the Social Sciences (SPSS), versão 18, foi utilizado, sendo descrita a frequência dos achados epidemiológicos. As médias e os desvios padrões das variáveis contínuas (idade e IMC) dos grupos de mulheres com gestações de baixo risco e perdas repetidas foram comparados utilizando-se o teste $t$ de Student, por apresentarem distribuição normal. As frequências das variáveis nominais foram comparadas aplicando-se o teste do $\chi^{2}$.

O projeto foi aprovado pelo Comitê de Ética em Pesquisa da na Maternidade Climério de Oliveira da Universidade Federal da Bahia, em 13 de setembro de 2012, com o n ${ }^{\circ}$ CAAE 06123412.0.0000.5543.

\section{Resultados}

A média de idade das mulheres com perdas foi superior às gestantes de baixo risco, sendo 32,3 e 26,5 respectivamente $(\mathrm{p}<0,01)$. O consumo de álcool, ainda que eventual, foi mais frequente nas mulheres com perdas gestacionais do que no Grupo Controle (36,9 versus $22,1 \% ; \mathrm{p}<0,01)$.

O IMC foi de $26,9 \mathrm{~kg} / \mathrm{m}^{2}$ entre as mulheres com perdas gestacionais e de $23,59 \mathrm{~kg} / \mathrm{m}^{2}$ nas gestantes de baixo risco $(\mathrm{p}<0,01)$. Quando agrupadas nas categorias de normal e sobrepeso/obesa, $60,7 \%$ do grupo de perdas e 31,9\% do Grupo Controle estavam no grupo de sobrepeso/obesa $(\mathrm{p}<0,01)$, como visto na Tabela 1 .

Quanto aos antecedentes obstétricos, 103 mulheres com perdas tiveram 332 gestações, sendo que uma mesma mulher, por vezes, apresentava várias perdas em idades gestacionais diferentes. Houve mais abortos no primeiro trimestre, e a sobrevida dos recém-nascidos das mulheres cujas gestações foram interrompidas no terceiro trimestre foi acentuadamente mais frequente (Tabela 2). Em 56 mulheres, predominaram 2 ou mais abortos do primeiro trimestre e, em 31 delas, 2 ou mais gestações foram interrompidas no segundo, configurando abortos tardios e recém-nascidos prematuros extremos. Um menor número de mulheres (21) evoluiu predominantemente para partos no terceiro trimestre da gestação. Quanto aos antecedentes no grupo das gestantes de baixo risco, 72 tiveram 92 gestações que

Tabela 1. Características epidemiológicas de mulheres com perdas gestacionais de repetição e gestantes de baixo risco (Grupo Controle)

\begin{tabular}{|c|c|c|c|}
\hline \multirow[t]{2}{*}{ Características } & $\begin{array}{c}\text { Mulheres com } \\
\text { perdas gestacionais }\end{array}$ & $\begin{array}{c}\text { Gestantes de } \\
\text { baixo risco }\end{array}$ & \multirow[t]{2}{*}{ Valor $p$} \\
\hline & $n(\%)$ & n (\%) & \\
\hline Idade (média e DP) & $32,3(6,3)$ & $26,5(6,4)$ & $<0,001$ \\
\hline \multicolumn{4}{|l|}{ Escolaridade } \\
\hline Fundamental & $28(27) 2$, & $61(29,9)$ & 0,8 \\
\hline Médio & $66(64,1)$ & $127(62,3)$ & \\
\hline Superior & $9(8,7)$ & $16(7,8)$ & 0,2 \\
\hline Ocupação com renda & $62(60,2)$ & $110(53,9)$ & \\
\hline Estado civil (casada/unic̃o estável) & $96(93,2)$ & $136(66,7)$ & $<0,001$ \\
\hline Etilismo & $38(36,9)$ & $45(22,1)$ & 0,006 \\
\hline \multicolumn{4}{|l|}{ Raça } \\
\hline Negra & $57(55,3)$ & $51(25,0)$ & $<0,001$ \\
\hline Parda & $38(36,9)$ & $122(59,8)$ & \\
\hline Branca & $8(7,8)$ & $31(15,2)$ & \\
\hline \multicolumn{4}{|l|}{ Classes de IMC* } \\
\hline Normal & $35(39,3)$ & $137(68,2)$ & $<0,001$ \\
\hline Sobrepeso & $28(31,5)$ & $49(24,4)$ & \\
\hline Obeso & $26(29,2)$ & $15(7,5)$ & \\
\hline
\end{tabular}

Estado civil - casadas e união estável foram somados, estão em oposição às solteiras; DP: desvio padrão; IMC: índice de massa corpórea; *normal: 18,5 a $24,9 \mathrm{~kg} / \mathrm{m}^{2}$; sobrepeso: 25 a $29,9 \mathrm{~kg} / \mathrm{m}^{2}$ e obeso maior que $30 \mathrm{~kg} / \mathrm{m}^{2} 26$. 
Tabela 2. Antecedentes obstétricos: término da gestaç̃o, nativivos e sobreviventes em mulheres com perdas gestacionais de repetição e gestantes de baixo risco

\begin{tabular}{lccccc}
\hline $\begin{array}{l}\text { Término da gestação } \\
\text { (em trimestre) }\end{array}$ & $\begin{array}{c}\text { Número de } \\
\text { mulheres }\end{array}$ & $\begin{array}{c}\text { Número de } \\
\text { gestações }\end{array}$ & $\begin{array}{c}\text { Número de } \\
\text { nativivos }\end{array}$ & $\begin{array}{c}\text { Sobreviventes } \\
(\%)\end{array}$ \\
\hline $\begin{array}{l}\text { Mulheres com perdas } \\
1^{\circ}\end{array}$ & 77 & 159 & - & - \\
$2^{\circ}$ & 49 & 93 & 52 & 5,7 \\
$3^{\circ}$ & 47 & 80 & 55 & 43,8 \\
Gestantes de baixo risco & & & & \\
Primigesta & 87 & - & - & - \\
$1^{\circ}$ & 72 & 92 & - & - \\
$2^{\circ}$ e $3^{\circ}$ & 88 & 168 & 162 & 98,1 \\
\hline
\end{tabular}

resultaram em abortamentos do primeiro trimestre e 88 tiveram seus recém-nascidos no segundo e terceiro trimestre. Desses, a maioria sobreviveu. Contudo, houve três óbitos neonatais, um por prematuridade e dois por malformação, além disso seis natimortos devido à pré-eclâmpsia, macrossomia fetal e causa desconhecida.

\section{Discussão}

Pretendeu-se com este trabalho descrever as características epidemiológicas e obstétricas de 103 mulheres atendidas num Ambulatório de Perdas Gestacionais de Repetição, em comparação com 204 gestantes de um pré-natal de baixo risco em uma maternidade pública de Salvador, na Bahia.

A idade das mulheres com perdas gestacionais foi superior àquelas das gestantes de baixo risco e essa diferença pode estar relacionada ao fato de que as mulheres com perdas, somente após duas ou mais tentativas sem êxito, são admitidas para investigação, enquanto muitas gestantes de baixo risco eram primigestas. Quando comparadas as amostras deste trabalho, verifica-se que 87/204 $(42,6 \%)$ dos controles eram primigestas, diferentemente de outros autores ${ }^{5,6,27}$, cujos controles tinham pelo menos um nascido vivo e não houve relato de diferença de idade entre os dois grupos, ou quando houve, era insignificante. A inclusão das primigestas, conquanto tenham evoluído na quase totalidade para partos de nativivos a termo, representa, porém, uma limitação deste estudo, comprometendo a comparação não somente da idade, mas de outros parâmetros pesquisados.

A predominância de mulheres casadas e em união estável no grupo com perdas gestacionais e a estabilidade emocional daí decorrente representam, provavelmente, fatores que favorecem o enfrentamento dos insucessos gestacionais e a disposição para novas tentativas. Trata-se de um perfil bem distinto do grupo de baixo risco, em que as mulheres eram mais jovens, solteiras e com gestações nem sempre planejadas.
Destacou-se na comparação dos dois grupos o peso corporal, sendo o IMC significativamente mais elevado entre mulheres com perdas gestacionais. A diferença pode estar relacionada ao número de jovens primigestas incluídas no Grupo Controle, não expostas à gestação, o que é um fator de risco reconhecido no desencadeamento e na manutenção da obesidade ${ }^{16}$. Um estudo com 433 puérperas, jovens e sem doenças crônicas, em uma maternidade pública do Rio de Janeiro, evidenciou 106 $(24,5 \%)$ delas com sobrepeso/obesidade, sendo a préeclâmpsia a intercorrência mais frequente, associada ao desvio ponderal ${ }^{20}$. Tais dados são concordantes com aqueles do grupo de baixo risco, embora inferiores aos $31,5 \%$ das mulheres com perdas recorrentes, nas quais a obesidade pode ser incluída entre os fatores que concorreram para o insucesso das gestações. Numa investigação de trombofilias hereditárias em mulheres com perdas de repetição, os autores ${ }^{15}$ excluem da casuística mulheres obesas, a fim de evitar um fator confundidor dos resultados. Em um trabalho ${ }^{22}$, com ênfase no futuro reprodutivo de mulheres com perdas gestacionais de repetição, evidenciou-se que aquelas com obesidade ou baixo peso apresentam maiores riscos para um aborto subsequente do que as normais e com sobrepeso. Um outro estudo ${ }^{21}$, que teve como objetivo verificar a associação entre IMC e gestação futura em 696 mulheres com perdas gestacionais de causas desconhecidas, revelou a obesidade como fator de risco independente para outro abortamento, acrescido pela idade materna e quantidade de abortos prévios. No presente material, aproximadamente um terço das mulheres com perdas está no grupo sobrepeso/ obesa, embora aquelas realmente obesas representem uma pequena proporção que, de acordo com a recomendação destes autores e outros serviços ${ }^{17}$, necessitam de ajuste ponderal na fase pré-concepcional, com alimentação adequada e exercícios físicos regulares para um aumento da chance do êxito gestacional.

Quanto aos antecedentes obstétricos, constatou-se que algumas mulheres relataram várias perdas em idades gestacionais diferentes, dado também assinalado por outros autores ${ }^{27-29}$. Ao investigarem trombofilias hereditárias, tais autores incluíram mulheres com passado de dois ou mais abortamentos precoces e tardios, além de óbitos fetais em outras fases da gestação. No presente trabalho, um maior número de mulheres relatou dois ou mais abortos no primeiro trimestre, seguido daquelas com abortamentos tardios ou prematuros extremos que, em sua maioria, evoluíram para o óbito fetal ou neonatal. A predominância de abortamentos no primeiro trimestre coincide com os dados de 334 mulheres com perdas recorrentes na gravidez, nas quais a maioria ocorreu antes da $14^{a}$ semana, principalmente na fase pré-embriônica ${ }^{6}$. Das 103 mulheres do grupo 
de perdas, 49 e 47 tiveram gestações interrompidas nos segundo e terceiro trimestres respectivamente, com frequentes relatos de herniação de bolsa amniótica, amniorrexe e trabalho de parto prematuro que podem corresponder a um processo contínuo com vários fatores associados ${ }^{30}$ levando ao nascimento de conceptos no limite da vitabilidade, época em que poderiam ter sido beneficiadas por intervenções médicas que resultassem no êxito na gestação.

Os resultados deste estudo revelam que mulheres com perdas gestacionais constituem um grupo heterogêneo e que a investigação criteriosa dos antecedentes obstétricos é de maior importância como o primeiro passo para elucidação diagnóstica e conduta.

\section{Referências}

1. Montenegro CAB, Rezende Filho J. Abortamento. In: Montenegro CA, Rezende Filho J. Rezende: obstetrícia fundamental. 11 ed. Rio de Janeiro: Guanabara Koogan; 2008. p. 218-33.

2. Ruisk K, Altman K. Perda gestacional recorrente. In: Fortner KB, Szymanski LM, Fox HE, Wallach EE, editores. Manual de ginecologia e obstetrícia do John Hopkins. 3 ed. São Paulo: Artmed; 2009. p. $468-72$.

3. Practice Committee of the American Society for Reproductive Medicine. Evaluation and treatment of recurrent pregnancy loss: a committee opinion. Fertil Steril. 2012;98(5):1 103-1 1 .

4. Hussein AS, Darwish H, Shelbayeh K. Association between factor V Leiden mutation and poor pregnancy outcomes among Palestinian women. Thromb Res. 2010;126(2):e78-82.

5. Serrano F, Lima ML, Lopes C, Almeida JP, Branco J. Factor V Leiden and prothrombin G20210A in Portuguese women with recurrent miscarriage: is it worthwhile to investigate? Arch Gynecol Obstet. $2011 ; 284(5): 1127-32$.

6. Heuser C, Dalton J, Macpherson C, Branch DW, Porter TF, Silver RM. Idiopathic recurrent pregnancy loss recurs at similar gestational ages. Am J Obstet Gynecol. 2010;203(4):343.e 1-5.

7. Practice Committee of the American Society for Reproductive Medicine. Definitions of infertility and recurrent pregnancy loss: a committee opinion. Fertil Steril. 2013;99(1):63.

8. Kutteh $W H$, Hinote $C D$. Antiphospholipid antibody syndrome. Obstet Gynecol Clin North Am. 2014;41(1):113-32.

9. Germeyer A, Savaris RF, Jauckus J, Lessey B. Endometrial beta3 Integrin profile reflects endometrial receptivity defects in women with unexplained recurrent pregnancy loss. Reprod Biol Endocrinol. 2014;12:53.

10. Bastos R, Ramalho C, Dória $S$. Estudo da prevalência de anomalias cromossómicas em abortamentos espontâneos ou mortes fetais. Acta Med Port. 2014;27(1):42-8.

11. Gonçalves RO, Santos WVB, Sarno M, Cerqueira BAV, Gonçalves MS, Costa OLN. Chromosomal abnormalities in couples with recurrent first trimester abortions. Rev Bras Ginecol Obstet. 2014;36(3):113-7.

12. Fraga LR, Dutra CG, Boquett JA, Vianna FS, Gonçalves RO, Paskulin DD, et al. p53 signaling pathway polymorphisms associated to pregnancy loss. Mol Biol Rep. 2014;41(3):1871-7.

13. Dutra CG, Fraga LR, Nácul AP, Passos EP, Gonçalves RO, Nunes OL, et al. Lack of association between thrombophilic gene variants and recurrent pregnancy loss. Hum Fertil (Camb). 2014;17(2):99-105.

14. Parveen F, Shukla A, Agrawal S. Should factor V Leiden mutation and prothrombin gene polymorphism testing be done in women with recurrent miscarriage from North India? Arch Gynecol Obstet. 2013;287(2):375-81

15. Bagheri M, Rad IA, Nanbakhsh F. Factor V Leiden G1691A and factor II G202 $10 A$ point mutations and pregnancy in North-West of Iran. Arch Gynecol Obstet. 2011 ;284(5):131 1-5.
16. Nogueira Al, Carreiro MP. Obesidade e gravidez. Rev Med Minas Gerais. 2013;23(1):88-98.

17. Van den Berg MM, Vissenberg R, Goddijn M. Recurrent miscarriage clinics. Obstet Gynecol Clin North Am. 2014;41(1):145-55.

18. Gonçalves CV, Mendoza-Sassi RA, Cesar JA, Castro NB, Bortolomedi AP. [Body mass index and gestational weight gain as factors predicting complications and pregnancy outcome]. Rev Bras Ginecol Obstet. 2012;34(7):304-9. Portuguese.

19. Santos EM, Amorim LP, Costa OL, Oliveira N, Guimarães AC. [Profile of gestational and metabolic risk in the prenatal care service of a public maternity in the Brazilian Northeast]. Rev Bras Ginecol Obstet. 2012;34(3):102-6. Portuguese.

20. Seabra G, Padilha PC, Queiroz JA, Saunders C. [Pregestational overweight and obesity: prevalence and outcome associated with pregnancy]. Rev Bras Ginecol Obstet. $2011 ; 33(11): 348-53$. Portuguese.

21. Lo W, Rai R, Hameed A, Brailsford SR, Al-Ghamdi AA, Reagan L. The effect of body mass index on the outcome of pregnancy in women with recurrent miscarriage. J Family Community Med. 2012;19(3): 167-71.

22. Metwally M, Saravelos SH, Ledger WL, Li TC. Body mass index and risk of miscarriage in women with recurrent miscarriage. Fertil Steril. 2010;94(1):290-5.

23. Miyakis S, Lockshin MD, Atsumi T, Branch DW, Brey RL, Cervera R, et al. International consensus statement on an update of the classification criteria for definite antiphospholipid syndrome (APS). J Thromb Haemost. 2006;4(2):295-306.

24. Christiansen $O B$. Research methodology in recurrent pregnancy loss. Obstet Gynecol Clin North Am. 2014;41(1):19-39.

25. Khan NA, Hemmelgarn B, Herman RJ, Rabkin SW, McAlister FA, Bell CM, et al. The 2008 Canadian Hypertension Education Program recommendations for the management of hypertension: part 2- therapy. Can J Cardiol. 2008;24(6):465-75.

26. Bhattacharya S, Campbell DM, Liston WA, Bhattacharya S. Effect of body mass index on pregnancy outcomes in nulliparous women delivering singleton babies. BMC Public Health. 2007;7:168.

27. Brenner B, Sarig G, Weiner Z, Younis J, Blumenfeld Z, Lanir N. Thrombophilic polymorphisms are common in women with fetal loss without apparent cause. Thromb Haemost. 1999;82(1):6-9.

28. Settin A, Alkasem R, Ali E, El Baz R, Mashaley AM. Factor V Leiden and prothrombin gene mutations in Egyptian cases with unexplained recurrent pregnancy loss. Hematology. $2011 ; 16(1): 59-63$.

29. Kovalevsky G, Gracia CR, Berlin JA, Sammel MD, Barnhart KT. Evaluation of the association between hereditary thrombophilias and recurrent pregnancy loss: a meta-analysis. Arch Intern Med. 2004; 164(5):558-63.

30. McNamee KM, Dawood F, Farquharson RG. Mid-trimester pregnancy loss. Obstet Gynecol Clin North Am. 2014;41(1):87-102. 